\section{Use of Flow Cytometric Methods to Quantify Protein-Protein Interactions}

\author{
Levi L. Blazer, ${ }^{1}$ David L. Roman, ${ }^{4}$ Molly R. Muxlow, ${ }^{1}$ and \\ Richard R. Neubig ${ }^{1,2,3}$
}

${ }^{1}$ Department of Pharmacology, The University of Michigan Medical School, Ann Arbor, Michigan

${ }^{2}$ Department of Internal Medicine, The University of Michigan Medical School, Ann Arbor, Michigan

${ }^{3}$ Center for Chemical Genomics, The University of Michigan Medical School, Ann Arbor, Michigan

${ }^{4}$ Division of Medicinal and Natural Products Chemistry, The University of Iowa, Iowa City, Iowa

\begin{abstract}
A method is described for the quantitative analysis of protein-protein interactions using the flow cytometry protein interaction assay (FCPIA). This method is based upon immobilizing protein on a polystyrene bead, incubating these beads with a fluorescently labeled binding partner, and assessing the sample for bead-associated fluorescence in a flow cytometer. This method can be used to calculate protein-protein interaction affinities or to perform competition experiments with unlabeled binding partners or small molecules. Examples described in this protocol highlight the use of this assay in the quantification of the affinity of binding partners of the regulator of G-protein signaling protein, RGS19, in either a saturation or a competition format. An adaptation of this method that is compatible for high-throughput screening is also provided. Curr. Protoc. Cytom. 51:13.11.1-13.11.15. (C) 2010 by John Wiley \& Sons, Inc.
\end{abstract}

Keywords: RGS • G protein $\bullet$ protein-protein interaction $\bullet$ FCPIA • high-throughput screening $\bullet$ multiplexing

\section{INTRODUCTION}

Nearly all biological processes utilize a protein-protein interaction (PPI) at some level. As our understanding of cellular processes evolves, it has become clear that a thorough understanding of how two or more proteins interact in a functional manner is a critical factor in determining the fundamental mechanisms governing cellular processes. Due to the importance of protein-protein interactions in biology, a number of methods have been developed to study the nature of these binding events in vitro. These methods fall generally into one of three classes: solution-phase methods [e.g., fluorescence polarization, intrinsic fluorescence changes, fluorescence resonance energy transfer (FRET)/fluorescence quenching, isothermal calorimetry, nuclear magnetic resonance (NMR)]; cell-based methods [e.g., bioluminescence resonance energy transfer (BRET) reporters, split luciferase reporters, yeast/mammalian two hybrid, beta-galactosidase complementation assays]; and solid-surface methods (e.g., co-immunoprecipitation, surface plasmon resonance). Many of these methods are based upon studying the interaction between two or more recombinantly expressed and purified proteins. These approaches, including the methodology being presented here, have proven to be extremely valuable tools in the study of PPIs. The method described here has significant advantages over these more traditional tools, especially for the quantification of PPI affinities and in the development of small molecule protein-protein interaction inhibitors.
Multiplexed and MicroparticleBased Analyses

\subsection{1}

Supplement 51 


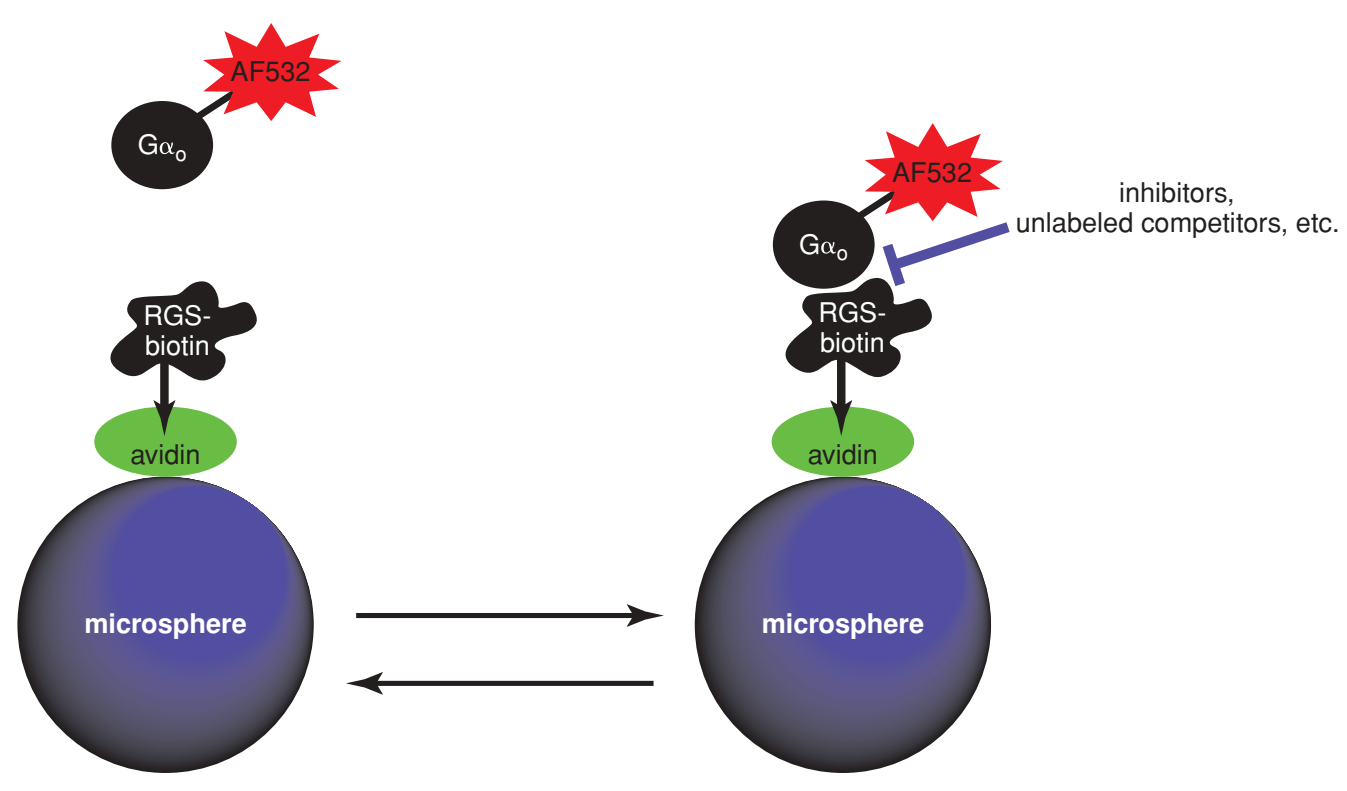

Figure 13.11.1 Schematic of FCPIA approach. Avidin-coated microspheres are labeled with biotinylated RGS proteins. The immobilized RGS proteins are incubated with Alexa Fluor-532-labeled $\mathrm{G} \alpha_{0}$. The ability of small molecules or competing unlabeled RGS proteins to inhibit this interaction can be characterized by their ability to diminish bead-associated Alexa Fluor-532 fluorescence. The assay can be expanded by labeling distinctly identifiable beads (e.g., Luminex bead regions) with different RGS proteins and performing the experiment in a multiplex format.

Flow Cytometry to Quantify Protein-Protein Interactions
This unit describes a method for the quantitative analysis of protein-protein interactions using the flow cytometry protein-protein interaction assay (FCPIA; Fig. 13.11.1). This methodology is based upon immobilizing a binding partner (target) to polystyrene beads and incubating these beads in a solution of a fluorescently labeled binding partner (reporter) in the presence or absence of competitors. This method can be used to characterize a variety of protein-protein interactions (Sarvazyan et al., 1998; Sklar et al., 2002; Simons et al., 2003) and to identify or characterize inhibitors of these binding events (Simons et al., 2003; Roman et al., 2007; Roof et al., 2008), even in a high throughput manner [see Roman et al. (2007) and Alternate Protocol 1]. This assay has been designed primarily for use with purified and chemically labeled proteins, but has also been adapted for use with cell lysates and other complex biological mixtures (Buranda et al., 2009).

The protein-protein interaction upon which most of our work with this protocol is based is that of the interaction between a regulator of G protein signaling (RGS) and a heterotrimeric $\mathrm{G}$ protein $\alpha$ subunit $(\mathrm{G} \alpha)$. RGS proteins are potent negative modulators of G protein signaling (Berman et al., 1996; Neubig and Siderovski, 2002; Blazer and Neubig, 2008). They function by binding to the active (GTP-bound) form of G $\alpha$ subunits and inducing a conformational change in the $\mathrm{G}$ protein that accelerates the rate of GTP hydrolysis (Berman et al., 1996). This interaction is heavily dependent upon the nucleotide-bound state of the G protein, whereby the RGS protein binds weakly $\left(K_{\mathrm{d}}>1 \mu \mathrm{M}\right)$ to the guanosine diphosphate (GDP) state, but binds with high affinity $\left(K_{\mathrm{d}} \sim 1\right.$ to $10 \mathrm{nM}$ ) to the GDP-aluminum fluoride state (Fig. 13.11.2). In this conformation, the aluminum fluoride complex sits in a planar configuration in the gamma phosphate-binding site on the $G$ protein. It has been proposed that this conformation is similar in nature to the transition state of GTP hydrolysis (Berman et al., 1996). The strong dependence on aluminum fluoride for the RGS-G $\alpha$ interaction will be used as a means to differentiate between specific and nonspecific binding for this particular PPI.

13.11.2 


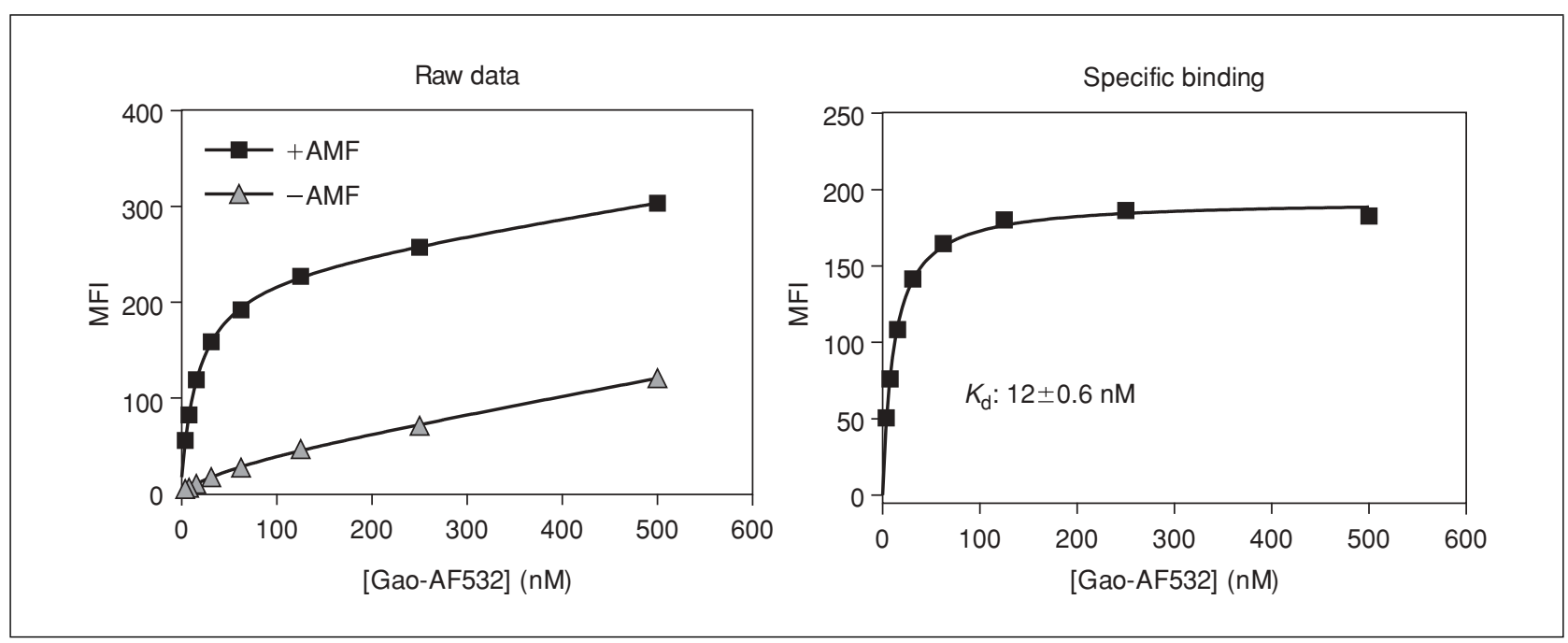

Figure 13.11.2 Saturation of RGS19 binding to $\mathrm{G} \alpha_{0}$ in the presence or absence of GDP, aluminum fluoride, and magnesium. Nonspecific binding is defined by residual affinity of RGS19 for G $\alpha_{0}$-GDP in the absence of aluminum fluoride (-AMF). As can be observed, the interaction between RGS19 and $G \alpha_{0}$ binding is dependent on aluminum fluoride and is of high affinity $\left(K_{\mathrm{d}} \sim 12 \mathrm{nM}\right)$.

This assay is applicable to the study of many PPIs other than the RGS-G $\alpha$ interaction. In the nervous system, PDZ domain containing proteins are important scaffolds for signaling systems, especially in the post-synaptic density. One particular PDZ domain containing protein, $\mathrm{G}$ alpha interacting protein C-terminus (GIPC), has been suggested to be important in localizing RGS19 to the D2 and D3, but not D4 dopamine receptor (Jeanneteau et al., 2004a,b). We have quantified the affinity of GIPC for RGS19 using this method (Fig. 13.11.3). Furthermore, we show that the affinity is independent of which protein is the "target" protein (e.g., the immobilized protein on the bead). To confirm that the nonstereotypical PDZ motif on the C-terminus of RGS19 (QSSEA) is the predominant motif required for the GIPC-RGS19 interaction, a mutant of RGS19 with a C-terminal truncation of the last 11 amino acids (including the PDZ ligand) was generated. This mutant protein is incapable of competing with wild-type RGS19 for binding to fluorescently labeled GIPC, suggesting that the PDZ motif is indeed necessary for the GIPC-RGS19 interaction. The observation that GIPC and RGS19 interact in a PDZ-dependent manner provides further evidence that GIPC may function as a scaffold for RGS19. For this set of experiments, a competition format was particularly useful. Unlike a saturation experiment, the competition experiment directly compares the ability of two proteins to compete for the binding of the reporter protein, as opposed to trying to measure weak or non-existent binding of a PDZ ligand-deficient RGS19 to GIPC.

This assay is also useful for the rapid screening of hybridoma clones in the development of monoclonal antibodies. This method is useful in clone screening because it is easily multiplexed, allowing for the study of the antigenic target and several related targets in a single well. The ability to screen for antigenic specificity earlier in the hybridoma screening reduces both the number of clones for follow up and the time required to identify the optimal clone. To prove this concept, we generated hybridomas from mice that had been immunized with RGS19 and screened these clones for the ability to selectively bind RGS19 over several other related RGS proteins in a multiplexed manner using $<50 \mu \mathrm{l}$ of culture supernatant (Fig. 13.11.4). This was performed by immobilizing RGS proteins or the fusion protein with which the antigen was originally expressed (maltose binding protein; MBP) on the bead. The beads were then incubated with a small volume of hybridoma supernatant and an excess of phycoerythrin (PE) labeled anti-mouse IgG. MBP was included as a control because the RGS19 was expressed and

Multiplexed and MicroparticleBased Analyses

\subsection{3}


A

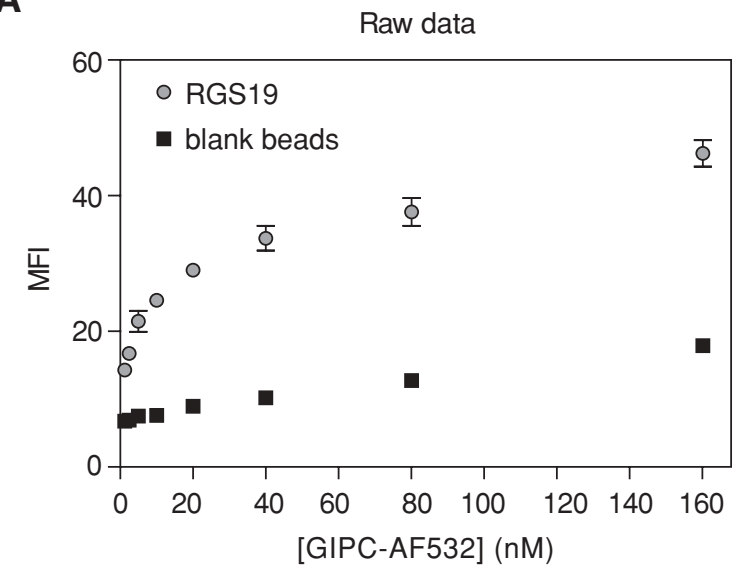

B

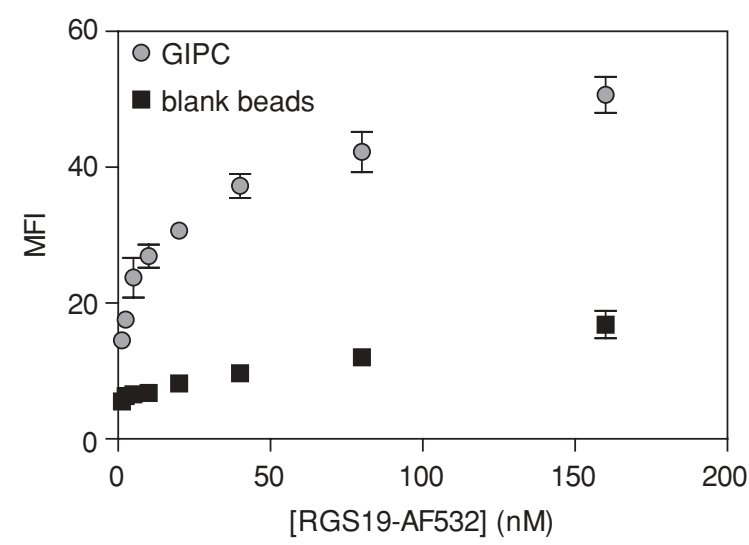

Specific binding

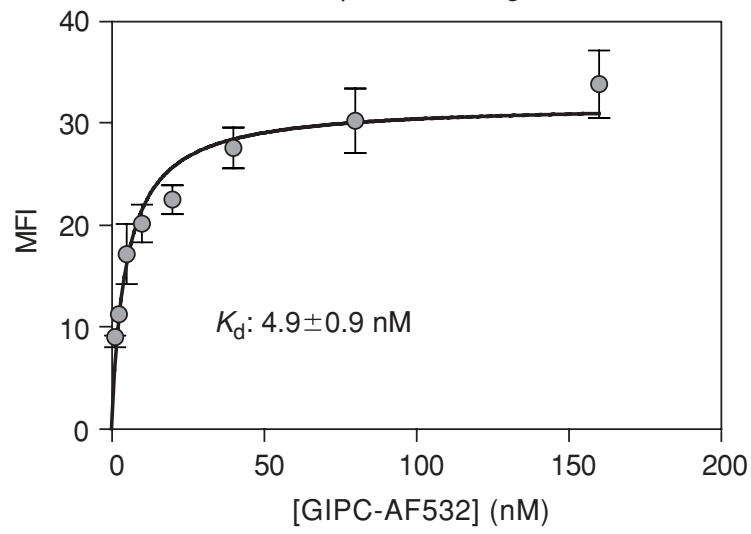

Specific binding

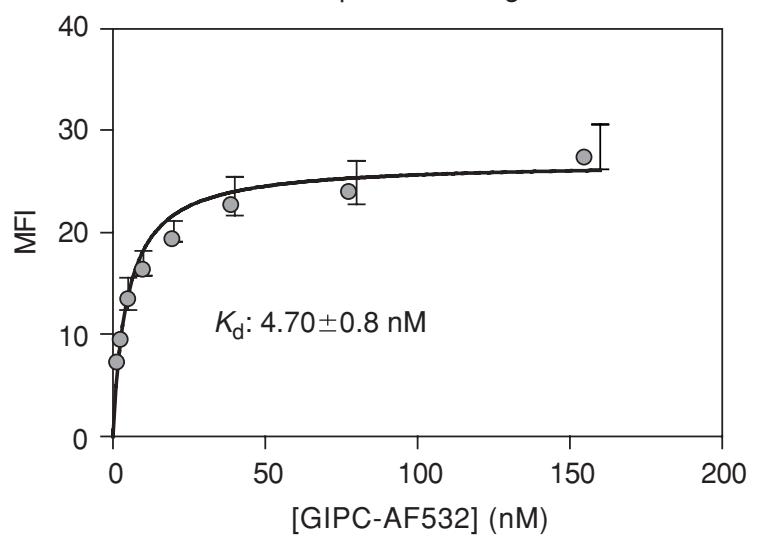

C

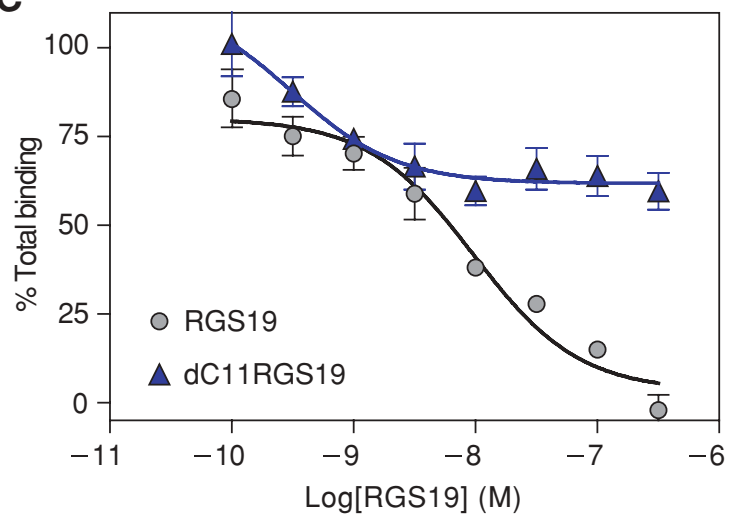

Figure 13.11.3 Characterization of the RGS19-GIPC protein-protein interaction. Saturation of RGS19 binding to GIPC is independent of which protein is immobilized on the bead. (A) Saturation of biotinylated RGS19 by Alexa Fluor-532-labeled GIPC. (B) Saturation of biotinylated GIPC by Alexa Fluor-532-labeled RGS19. Notice that there is no difference in $K_{\mathrm{d}}$ or Bmax. (C) Immobilized wild-type RGS19 is competed by unlabeled wild-type but not $\triangle$ PDZ RGS19 (dc11RGS19) for binding to Alexa Fluor-532-labeled GIPC. Deletion of the PDZ domain abolishes the affinity of the wild-type RGS19.

Flow Cytometry

to Quantify

Protein-Protein

Interactions

13.11.4 


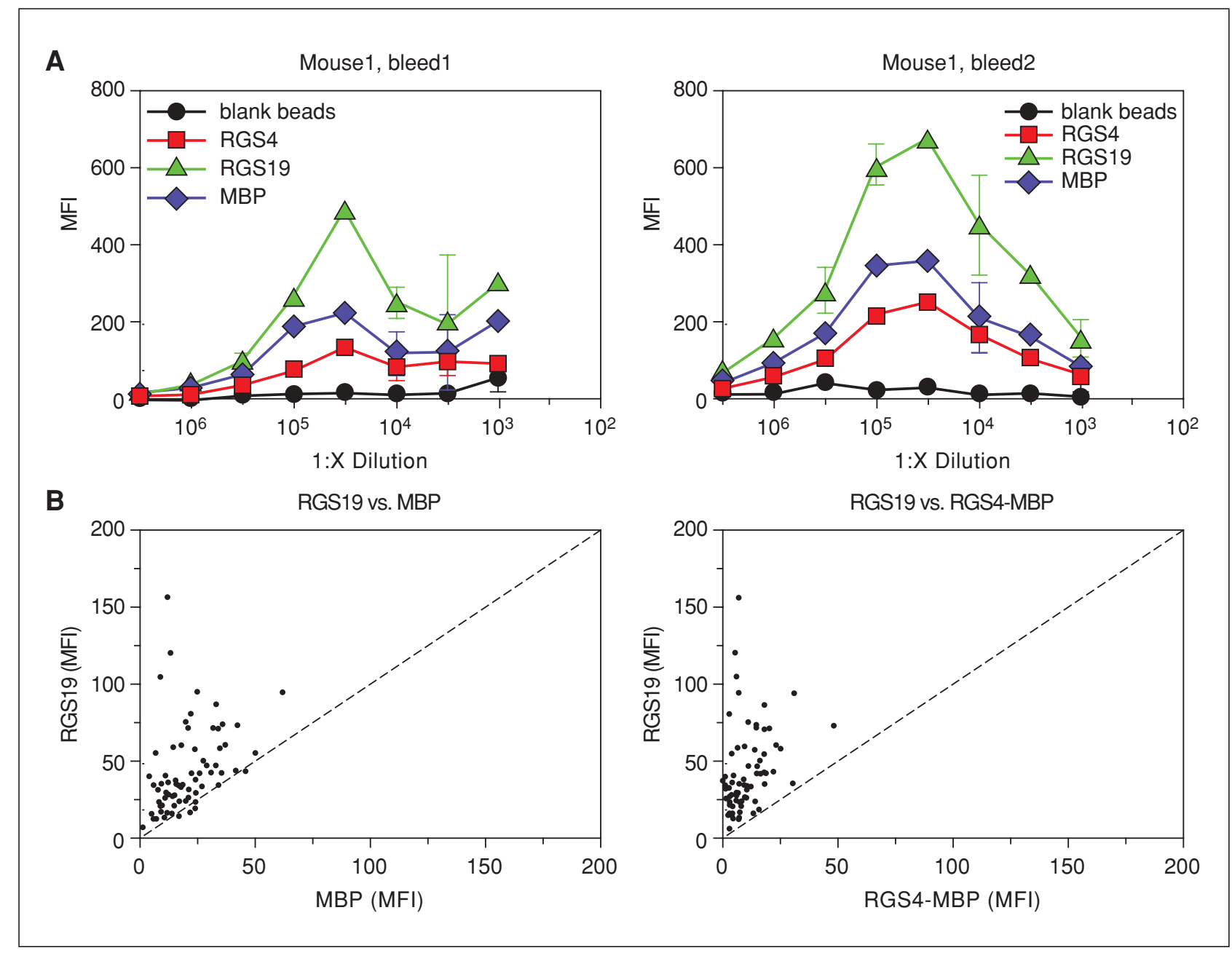

Figure 13.11.4 Multiplexed flow cytometry analysis during monoclonal antibody development. (A) Multiplexed titer analysis of sera from a representative mouse challenged with RGS19. (B) Representative single-point multiplexed hybridoma screening on 74 clones derived from the mouse in panel A. Notice the high specificity for the target antigen (RGS19) over MBP or RGS4-MBP fusion protein. This method allows a large number of hybridomas $(>200)$ to be screened in a single day for antigenic specificity.

purified as an MBP fusion protein. The MBP tag was proteolytically cleaved from the RGS and purified away before mice were immunized, but there was still the potential for low levels of contamination of the antigen with this bacterial protein.

The FCPIA methodology described in this unit is applicable to the study of many proteinprotein interactions. It is amenable to the multiplexing of several target proteins for the binding to a single reporter, saving both time and reagents. The main limitation of this approach is the apparent affinity limitations that we have observed. PPIs with $K_{\mathrm{d}}$ values weaker than $1 \mu \mathrm{M}$ or so have been difficult to resolve using this method.

\section{CHARACTERIZING PROTEIN-PROTEIN BINDING USING THE FLOW CYTOMETRY PROTEIN-PROTEIN INTERACTION ASSAY}

Avidin-coated polystyrene beads are saturated with a purified and biotinylated binding partner and incubated in varying amounts with a purified Alexa Fluor-532-labeled binding partner. After equilibration, bead-associated fluorescence is measured by flow cytometry. This application is easily multiplexed by using optically encoded beads, which are commercially available from a variety of sources, and a suitable flow cytometer (reviewed in UNIT 13.8), allowing for simultaneous detection of reporter protein interactions with multiple targets.

Multiplexed and MicroparticleBased Analyses

\subsection{5}


BASIC PROTOCOL 1
Flow Cytometry to Quantify Protein-Protein Interactions

\section{SATURATION ANALYSIS OF BIOTIN-RGS WITH ALEXA FLUOR532-G $\alpha_{0}$}

This protocol is designed to measure the saturatable, aluminum fluoride-dependent binding of $\mathrm{G} \alpha_{\mathrm{o}}$ to RGS4. This assay requires 32 wells on a 96-well plate, 16 for dilutions of $G \alpha_{0}$ in the presence of AMF and 16 for dilutions of $G \alpha_{0}$ in the absence of AMF. The latter provides a measure of nonspecific binding and it is considered background (Roman at al., 2007; Shankaranarayanan et al., 2008).

\section{Materials}

LumAvidin polystyrene beads (L100-Lxxx-01, where the "xxx" represents the bead region)

Bead coupling buffer (BCB; see recipe)

Biotinylated RGS protein (see Support Protocol 1)

Flow buffer (FB; see recipe)

Alexa Fluor532-conjugated $\mathrm{G} \alpha_{\mathrm{o}}$ (see Support Protocol 2)

$50 \mu \mathrm{M}$ aluminum chloride, aqueous

$50 \mathrm{mM}$ magnesium chloride, aqueous

$50 \mathrm{mM}$ sodium fluoride, aqueous

1.5-ml microcentrifuge tubes

Microcentrifuge, preferably fixed-angle rotor

Vortex

Full-skirted 96-well polypropylene PCR plate (ISC Bioexpress, cat. no. T-3082-1)

Multichannel pipet

Aluminum foil

Luminex 200 flow cytometer (Luminex)

1. Add 60,000 LumAvidin beads to $1 \mathrm{ml}$ of $\mathrm{BCB}$ in a $1.5-\mathrm{ml}$ microcentrifuge tube. Pellet the beads by centrifuging $1 \mathrm{~min}$ at $5000 \times \mathrm{g}, 4^{\circ} \mathrm{C}$, in a microcentrifuge. Remove the supernatant and resuspend the pellet in $1 \mathrm{ml} \mathrm{BCB}$. Repeat this washing procedure with $1 \mathrm{ml}$ of $\mathrm{BCB}$ for a total of three washes.

As with most bead-based assays, it is important not to disrupt the pellet during aspiration of the supernatant.

2. Resuspend the beads in $200 \mu \mathrm{BCB}$ and vortex to disperse the pellet.

If necessary, light sonication in a bath sonicator may be used to ensure a monodisperse population of beads.

3. Add 25 to 100 pmol of biotinylated RGS protein to the beads and incubate $30 \mathrm{~min}$ at room temperature in the dark, mixing occasionally.

Gentle rotation or rocking may assist in bead coupling.

Since the bead binding capacity is very low $(\sim f m o l)$, the total amount of bead-bound target protein in the well will be very low compared to that of $G \alpha_{o}$, justifying the assumptions required for the standard nonlinear saturation curve analysis using total added ligand, even at low concentrations of $G \alpha_{o}$.

4. Wash the beads three times with BCB (see step 1) to remove nonspecifically bound target. Resuspend the beads in $2 \mathrm{ml} \mathrm{FB}$ and disperse the beads by vortexing.

5. Prepare the Alexa Fluor532-G $\alpha_{\mathrm{o}}$ working dilutions by diluting the stock to $2 \mu \mathrm{M}$ in $250 \mu \mathrm{l}$ of $\mathrm{FB}$ or $250 \mu \mathrm{l}$ of $\mathrm{FB}+5 \mu \mathrm{M} \mathrm{AlCl}_{3}, 5 \mathrm{mM} \mathrm{NaF}, 5 \mathrm{mM} \mathrm{MgCl} \mathrm{M}_{2}$ (AMF). Allow the $\mathrm{G} \alpha_{\mathrm{o}}$ to incubate for $10 \mathrm{~min}$ at room temperature to allow full incorporation of aluminum fluoride into the protein.

The solubility of these salts is limited in $F B$; therefore, it is important to prepare a fresh dilution of salts from a stock solution in water for each experiment. 
Alexa Fluor532-G $\alpha_{o}$ is light sensitive. It is important to limit the light exposure of the working and stock solutions using opaque tubes and prudent laboratory technique.

6. Add $100 \mu \mathrm{l}$ aluminum fluoride-activated Alexa Fluor532-G $\alpha_{0}$ to well A1 and A2 of a full-skirted PCR plate. Add $100 \mu$ of unactivated Alexa Fluor532-G $\alpha_{0}$ to well A3 and A4.

7. Add $50 \mu \mathrm{l}$ of $\mathrm{FB}+\mathrm{AMF}$ to wells B1-H1 and B2-H2. Add $50 \mu \mathrm{l}$ of FB without AMF to wells B3-H3 and B4-H4.

8. Serially dilute the Alexa Fluor532-G $\alpha_{0}$ down the columns of the plate with a multichannel pipet by transferring $50 \mu \mathrm{l}$ of the solution from the previous well and thoroughly mixing. All wells should be left with $50 \mu \mathrm{l}$ of solution with two-fold serial dilutions. Discard the remaining $50 \mu \mathrm{l}$.

9. Add $50 \mu \mathrm{l}$ of the beads to all wells in columns 1 to 4 of the assay plate.

The final volume in the well should be $100 \mu l$, making the highest concentration of $G \alpha_{o}$ in the assay $1 \mu M$. For PPIs with weak affinity, this maximal concentration can be changed as needed, though much lower affinity interactions (e.g., $K_{d}>1$ to $\left.10 \mu \mathrm{M}\right)$ may be difficult to detect by FCPIA.

10. Cover the plate with aluminum foil and incubate $30 \mathrm{~min}$ at room temperature.

11. Analyze the plate in the Luminex 200 flow cytometer, collecting at least 100 events per well. For analysis, data should be consolidated into median fluorescence values for each bead set per well.

This assay is compatible with any standard flow cytometer. The Luminex instrument provides the advantage of having predefined gating regions for their proprietary beadsets. If a standard cytometer is used, it is important to use appropriate gating to ensure that only data from monodisperse beads are included.

\section{FCPIA COMPETITION ASSAY}

It is often desirable to study the inhibition of a PPI by small molecules or competing proteins. This protocol provides a method to determine the inhibitory activity of a competitor (small molecule or protein) on the formation of the $\mathrm{G} \alpha_{0}$-RGS PPI. This method can be easily modified for single point high-throughput screening (Roman et al., 2007, 2009; also see Basic Protocol 3).

\section{Materials}

LumAvidin polystyrene beads (L100-Lxxx-01, where the "xxx" represents the bead region)

Bead coupling buffer (BCB; see recipe)

Biotinylated RGS protein (see Support Protocol 1)

Flow buffer (FB; see recipe)

Compound or competing protein (e.g., unlabeled RGS protein)

Alexa Fluor532-conjugated $\mathrm{G} \alpha_{\mathrm{o}}$ (see Support Protocol 2)

$50 \mu \mathrm{M}$ aluminum chloride, aqueous

$50 \mathrm{mM}$ magnesium chloride, aqueous

$50 \mathrm{mM}$ sodium fluoride, aqueous

1.5-ml microcentrifuge tubes

Microcentrifuge, preferably fixed-angle rotor

Vortex

Multichannel pipette or Multidrop micro reagent dispenser (Thermo-Fisher)

Luminex 200 flow cytometer (Luminex)

BASIC

PROTOCOL 2

Multiplexed and

Microparticle-

Based Analyses

13.11.7 
1. Wash 150,000 LumAvidin beads three times, each time with $1 \mathrm{ml}$ of BCB in a 1.5-ml microcentrifuge tube. Pellet the beads by centrifuging $1 \mathrm{~min}$ at $5000 \times \mathrm{g}, 4^{\circ} \mathrm{C}$, in a microcentrifuge. Resuspend the beads in $200 \mu \mathrm{BCB}$ and vortex to ensure a monodisperse population of beads.

This amount of beads provides enough labeled beads to fill a whole 96-well plate. It is often sufficient to use between 1000 to 3000 beads per well, so this assay can be scaled accordingly to your needs.

Light sonication may also assist in dispersing beads.

2. Add 50 to 200 pmol biotinylated RGS protein to the bead solution, mix, and incubate for $30 \mathrm{~min}$ at room temperature protected from light. Mix occasionally throughout the incubation.

3. Wash the beads three times, each time with $1 \mathrm{ml} \mathrm{BCB}$ (see step 1) and resuspend the pellet in $2 \mathrm{ml} \mathrm{FB}$.

4. Add $50 \mu \mathrm{l}$ of FB to all wells on the plate except for row A and row $\mathrm{H}$.

5. Dilute the compound or competing protein to $2 \times$ the final desired highest concentration in the serial dilution series. Make $200 \mu \mathrm{l}$ of this solution.

When testing a small molecule inhibitor in the RGS-G $\alpha_{o}$ FCPIA assay, the highest concentration of compound used is often determined by its solubility. If the competitor is an unlabeled RGS protein, a top concentration of $1 \mu \mathrm{M}$ is sufficient due to the high affinity $\left(K_{d} \sim n M\right)$ of $R G S$ proteins for $G \alpha_{o}-G D P / A M F$.

6. Serially dilute the working dilution of the compound or competing protein down the plate in $1 / 2 \log$ steps by adding $73.1 \mu \mathrm{l}$ of the diluted competitor to the wells in row A. Transfer $23.1 \mu$ down the plate through row G, mixing thoroughly. Discard the remaining $23.1 \mu \mathrm{l}$. Reserve the bottom row of the plate for positive and negative controls.

Usually duplicate samples are sufficient, allowing up to six competitors to be tested per 96-well plate.

7. Add an excess of competitor (or some known inhibitor) to six of the twelve wells in row $\mathrm{H}$. This will be your positive control. For a negative control (e.g., total binding), add vehicle to the other six wells.

8. Using a multichannel pipet or multidrop liquid handling unit, add $20 \mu \mathrm{l}$ of the beads to every well of the plate, including control wells. This will provide 1500 beads per well.

If a pretreatment of the RGS with compound is desired, a 15-min incubation may be added at this point.

9. Dilute Alexa Fluor532-G $\alpha_{\mathrm{o}}$ stock to $30 \mathrm{nM}$ in a final volume of $\mathrm{FB}+\mathrm{AMF}$. Allow the $\mathrm{G} \alpha_{\mathrm{o}}$ to activate for $10 \mathrm{~min}$ at room temperature in the dark.

Depending on the affinity of your PPI and the activity of your proteins, this concentration may need to be varied. Based upon your saturation data (see Basic Protocol 1), it is preferable to choose a final concentration of the $G \alpha_{o}$ that provides between $50 \%$ and $90 \%$ of the maximal response (Bmax) in a saturation assay.

10. Add $30 \mu \mathrm{l}$ of the activated AlexaFluor532-G $\alpha_{\mathrm{o}}$ (from step 9) to every well on the plate using a multichannel pipet or multidrop liquid handling unit. Incubate $30 \mathrm{~min}$ at room temperature, shielded from light.

Flow Cytometry to Quantify Protein-Protein Interactions

13.11.8

11. Analyze the experiment on a Luminex 200 flow cytometer, collecting at least 100 events/well. 
This protocol is a single-point version of the competition experiment described in Basic

Protocol 2 that has been optimized for high-throughput screening. This method has been scaled to a 384-well format and, like all protocols described in this unit, is amenable to multiplexing. Using a method very similar to the one presented here, we have screened over 200,000 small molecules using several multiplexed assays to identify inhibitors of $\mathrm{G} \alpha_{\mathrm{o}}$ binding to RGS4, RGS19, RGS16, RGS7, and RGS8 both in our laboratory, in collaboration with the Center for Chemical Genomics at the University of Michigan and in collaboration with the Molecular Library Probe Screening Network (MLPCN) at the University of New Mexico (Roman et al., 2007, 2009; MLPCN screening IDs: 1423, $1415,1439-41)$. For simplicity, this assay has been scaled to ten whole 384-well plates. The reader will notice that the fluorescent label on the $\mathrm{G} \alpha_{\mathrm{o}}$ has been changed to Alexa Fluor-488 for this application. The sole purpose for this was to allow for data acquisition using the standard blue laser available in most cytometers.

\section{Materials}

SPHERO streptavidin-coated particles, 5- to 5.9- $\mu \mathrm{m}$ diameter (Spherotech, SVP-50-5)

Bead coupling buffer (BCB; see recipe)

Biotinylated RGS protein (see Support Protocol 1)

Flow buffer (FB; see recipe)

Test or library compounds

Alexa Fluor-532-conjugated $\mathrm{G} \alpha_{\mathrm{o}}$ (see Support Protocol 2)

$50 \mu \mathrm{M}$ aluminum chloride, aqueous

$50 \mathrm{mM}$ magnesium chloride, aqueous

$50 \mathrm{mM}$ sodium fluoride, aqueous

1.5 -ml microcentrifuge tubes

Microcentrifuge, preferably with a fixed-angle rotor

Vortex mixer

Multidrop micro reagent dispenser (Thermo-Fisher)

Low volume, nonstick, black 384-well plate (Corning, cat. no. 3676)

HyperCyt liquid sampling unit (IntelliCyt)

Accuri C6 cytometer (Accuri Cytometers)

HyperView software

1. Wash $4.24 \times 10^{7}$ SPHERO beads three times, each time with $1 \mathrm{ml}$ of BCB in a $1.5-\mathrm{ml}$ microcentrifuge tube. Pellet the beads by centrifuging $1 \mathrm{~min}$ at $5000 \times g$, $4^{\circ} \mathrm{C}$, in a microcentrifuge. Resuspend the beads in $1.5 \mathrm{ml}$ of $\mathrm{BCB}$ and vortex to ensure a monodisperse population of beads.

This amount of beads provides enough labeled beads to fill ten 384-well plates at 5000 beads per well with several milliliters excess for the dead volume in the Multidrop micro reagent dispenser.

2. Add 250 pmol of biotinylated RGS protein to the bead population and incubate 30 min at ambient temperature.

Since the SPHERO beads tend to sink more rapidly than the LumAvidin beads used in the previous protocols, it is advisable to gently mix the beads several times during the labeling process.

3. Wash beads three times, each time in $1 \mathrm{ml} \mathrm{BCB}$ and resuspend the beads in $42 \mathrm{ml}$ FB.

This dilution will provide 5000 beads/10 $\mu \mathrm{l}$.

Multiplexed and MicroparticleBased Analyses

\subsection{9}


4. Using the Multidrop micro reagent dispenser, dispense $10 \mu \mathrm{l}$ of the beads into every well of a 384-well plate.

5. Add test or library compounds at desired concentration to columns 3 to 22 . Allow the compounds to incubate with the protein-bound beads for $30 \mathrm{~min}$.

Columns 1 and 2 are reserved for positive control wells ( $G \alpha_{o}$ in the absence of $\left.A M F\right)$.

Columns 23 and 24 are reserved as negative control wells ( $G \alpha_{o}$ in the presence of $\left.A M F\right)$.

The DMSO concentration should not exceed $2 \%$ of the assay volume.

6. Activate the $\mathrm{G} \alpha_{\mathrm{o}}$ by adding $2.34 \mathrm{nmol} \mathrm{G} \alpha_{\mathrm{o}}$ to $45 \mathrm{ml}$ of $\mathrm{FB}+\mathrm{AMF}$. For the positive control, add 240 pmol $G \alpha_{o}$ to $8 \mathrm{ml} \mathrm{FB}$ without AMF. Incubate $G \alpha_{0}$ in the dark for 10 min before use to allow AMF incorporation.

While not commonly observed, loss of the G $\alpha_{o}$ binding signal can be observed over time. If this occurs, multiple small batches of activated $G \alpha_{o}$ (e.g., enough for 2 to 5 plates) can be made.

7. Using the Multidrop micro reagent dispenser, add $10 \mu \mathrm{l}$ of activated $\mathrm{G} \alpha_{\mathrm{o}}$ in FB without AMF to columns 1 and 2 for the positive control. Add $10 \mu \mathrm{l}$ of the activated $\mathrm{G} \alpha_{\mathrm{o}}$ in $\mathrm{FB}+\mathrm{AMF}$ to the remaining columns (3 to 24). Allow the plates to incubate $40 \mathrm{~min}$ at room temperature in the dark.

8. Analyze the plate by aspirating samples through the HyperCyt liquid sampling unit and measuring the bead-associated fluorescence with the Accuri C6 flow cytometer.

An entire plate is sampled and analyzed in one continuous acquisition and the data is exported in one file to be analyzed by the HyperView software.

Since the HyperCyt automated liquid sampler continuously aspirates during collection, samples from every well are separated by the air bubble that forms during probe movement between wells. As these samples are fed into the flow cytometer, bursts of bead events are observed and can be correlated to a particular well from the sample plate.

The settings for both instruments (sip time, pump speed, flow rate, etc.) need to be optimized prior to analysis to ensure proper sample separation through the tubing from the liquid handling unit to the flow cytometer.

9. View and analyze the data using the HyperView software.

The raw data should be gated on forward/side scattering to eliminate bead aggregates and to identify bead regions. The number of events within the gate is plotted against acquisition time to apply bins to define each of the 384 wells. The median FL1-H value, corresponding to the amount of bound Alexa Fluor- $488 \mathrm{G} \alpha_{o}$, for each well is calculated by the software based on the bins determined.

The binning can potentially be double-checked by observing the extra time gap at the end of a column or row depending on the set-up of the sampling order of the plate wells. Different sampling templates are available, as well as options for a rinse after a desired number of samples through the HyperView software. This provides further confidence in correct binning and less carryover between wells.

10. Compare individual compound data points to the positive and negative control wells to calculate a percent inhibition. After screening the small molecule library, select potential lead compounds for follow-up dose response experiments based upon this percent inhibition value.

An alternative approach to identifying lead compounds is to use the number of standard deviations a test compound is away from the negative control as a measure of inhibitory activity. 
This protocol is a general method for biotinylating RGS proteins, although it is generally applicable to most purified protein samples that contain solvent exposed primary amines (e.g., lysine residues or unmodified $\mathrm{N}$-terminus).

\section{Materials}

1 to $10 \mathrm{mg}$ of purified protein, preferably $>90 \%$ homogeneous

Reaction buffer: $50 \mathrm{mM}$ HEPES, pH 9.0, at $4^{\circ} \mathrm{C}, 250 \mathrm{mM} \mathrm{NaCl}, 5 \%$ glycerol supplemented with $1 \mathrm{mM}$ tris(2-carboxyethyl)phosphine (TCEP)

$N$-hydroxysuccidimyl ester-biotin (NHS-biotin; Sigma-Aldrich, cat. no. B2643)

Dimethyl sulfoxide (DMSO)

$1 \mathrm{M}$ glycine (MP Biomedicals, cat. no. 808831) in $1 \mathrm{M}$ HEPES, pH 9.0, at $4^{\circ} \mathrm{C}$

Storage buffer: $50 \mathrm{mM}$ HEPES, $\mathrm{pH} 7.4$, at $4^{\circ} \mathrm{C}, 250 \mathrm{mM} \mathrm{NaCl}, 5 \%$ glycerol supplemented with $1 \mathrm{mM}$ tris(2-carboxyethyl)phosphine (TCEP)

10-ml Sephadex G25 gel filtration column (essentially any standard desalting column can be used)

Amicon Ultra centrifugal concentrators (MWCO, 10,000; Millipore, cat. no. UFC901096)

1. Exchange protein into reaction buffer by gel filtration.

This is a critical step, especially if the buffer in which the protein is stored contains primary amines (e.g., TRIS buffers), which will compete with the protein for the label.

Most RGS proteins are readily obtained through recombinant expression in E. coli (Berman et al., 1996; Roof et al., 2006; Roman et al., 2007). The only notable exception to this is RGS9.

2. Concentrate the protein, if necessary, to 2 to $8 \mathrm{mg} / \mathrm{ml}$.

Concentrate the protein using an Amicon Ultra centrifugal concentrator, per the manufacturer's instructions.

3. Dissolve $1 \mathrm{mg}$ of NHS-biotin to $50 \mathrm{mM}$ in DMSO.

4. Add a 3- to 5-fold molar excess of the NHS-biotin to the protein solution, being sure to add the DMSO slowly while swirling the tube.

This ensures that no local precipitation of the protein occurs upon addition of the organic solvent.

It is important to keep the DSMO concentration below $2 \%$ to ensure protein stability.

5. Allow the reaction to proceed $2 \mathrm{hr}$ at $4^{\circ} \mathrm{C}$ in the dark while gently shaking.

6. Quench the reaction by adding an excess of glycine to the tube.

A 5- to 10-fold molar excess of glycine to label is sufficient.

7. Purify the protein by gel filtration into storage buffer.

8. Concentrate protein to $>30 \mu \mathrm{M}$ using an Amicon Ultra centrifugal concentrator and store in small aliquots $(10 \mu \mathrm{l})$ at $-80^{\circ} \mathrm{C}$.

9. Test the function of labeled RGS protein.

This may be done by directly testing the ability of the labeled RGS to bind $G \alpha_{o}$ using Basic Protocol 1 or by using the single turnover GAP assay as described (Roof et al., 2006). 
SUPPORT PROTOCOL 2
Flow Cytometry to Quantify Protein-Protein Interactions 13.11.12

\section{ALEXA FLUOR532 LABELING OF G $\alpha_{0}$}

This protocol is a general method for fluorescently labeling $\mathrm{G} \alpha_{0}$, although it is generally applicable to most purified protein samples that contain solvent exposed thiols (e.g., cysteine residues). This protocol is identical for labeling $\mathrm{G} \alpha_{\mathrm{o}}$ with Alexa Fluor488.

\section{Materials}

1 to $10 \mathrm{mg}$ of purified $\mathrm{G} \alpha_{\mathrm{o}}$, preferably $>90 \%$ homogeneous

Tris(2-carboxyethyl)phosphine (TCEP)

Reaction buffer: $50 \mathrm{mM}$ HEPES, pH 7.4, at $4^{\circ} \mathrm{C}, 250 \mathrm{mM} \mathrm{NaCl}, 5 \%$ glycerol, supplemented with $10 \mu \mathrm{M}$ guanosine diphosphate (GDP)

AlexaFluor532 maleimide, $1 \mathrm{mg}$ (Invitrogen, cat. no. A10255)

Dimethyl sulfoxide (DMSO)

$1 \mathrm{M}$ dithiothreitol (powdered DTT), aqueous

Storage buffer: $50 \mathrm{mM}$ HEPES, pH 7.4, at $4{ }^{\circ} \mathrm{C}, 250 \mathrm{mM} \mathrm{NaCl}, 5 \%$ glycerol supplemented with $1 \mathrm{mM}$ TCEP and $10 \mu \mathrm{M}$ GDP

Amicon ultra centrifugal concentrators (MWCO, 10,000; Millipore, cat. no. UFC901096)

$10 \mathrm{ml}$ Sephadex G25 gel filtration column (essentially any standard desalting column can be used)

Spectrophotometer

1. Treat protein with a ten-fold molar excess of TCEP for 10 min on ice to ensure complete reduction of solvent accessible thiols.

$G \alpha_{o}$ is readily obtained from recombinant expression in $\mathrm{E}$. coli (Lee et al., 1994).

2. Exchange protein into reaction buffer by gel filtration.

3. Concentrate the protein, if necessary, to 2 to $8 \mathrm{mg} / \mathrm{ml}$.

Concentrate the protein using an Amicon Ultra centrifugal concentrator, per the manufacturer's instructions.

4. Dissolve $1 \mathrm{mg}$ of Alexa Fluor-532 maleimide to $20 \mathrm{mM}$ in DMSO.

5. Add a 3- to 5-fold molar excess of the Alexa Fluor-532 maleimide to the protein solution, being sure to add the DMSO slowly while swirling the tube.

This ensures that no local precipitation of the protein occurs upon addition of the organic solvent.

It is important to keep the DSMO concentration below $2 \%$ final concentration to ensure protein stability.

This labeling could also be performed on primary amines (e.g., $\varepsilon$-amines on lysine and free N-termini) using an amine reactive fluor, but GDP should be removed from the buffer. Removal of GDP will affect $G \alpha_{o}$ stability, so thiol labeling is recommended.

6. Allow the reaction to proceed $2 \mathrm{hr}$ at $4^{\circ} \mathrm{C}$ in the dark while gently shaking.

7. Quench the reaction by adding an excess of dithiothreitol to the tube.

A 5- to 10-fold molar excess of DTT to label is sufficient.

8. Purify the protein by gel filtration into storage buffer.

9. Concentrate protein to $>30 \mu \mathrm{M}$ using an Amicon ultra centrifugal concentrator. Store in $10-\mu 1$ aliquots up to 1 year at $-80^{\circ} \mathrm{C}$.

10. Determine the activity and concentration of $\mathrm{G} \alpha_{\mathrm{o}}$ by $\left[{ }^{35} \mathrm{~S}\right] \mathrm{GTP} \gamma \mathrm{S}$ binding and/or by spectrophotometric analysis $\left(\mathrm{A}_{280}\right)$.

Determining label incorporation can also be done spectrophotometrically, per the product insert provided with the Alexa Fluor-532 label. 
REAGENTS AND SOLUTIONS

Use deionized, distilled water in all recipes and protocol steps. For common stock solutions, see APPENDIX 2A; for suppliers, see SUPPLIERS APPENDIX.

\section{Bead coupling buffer (BCB)}

Phosphate-buffered saline (PBS; APPENDIX 2A), pH 7.4, supplemented with $1 \%$ bovine serum albumin (BSA) and sterile filtered through a $0.22-\mu \mathrm{m}$ filter to remove particulates that might be detected in the flow cytometer. Store in 50-ml aliquots up to 6 months at $-20^{\circ} \mathrm{C}$.

\section{Flow buffer (FB)}

Combine $50 \mathrm{mM}$ HEPES, $\mathrm{pH} 8.0$, at ambient temperature (pH adjusted with $10 \mathrm{~N}$ $\mathrm{NaOH}), 100 \mathrm{mM} \mathrm{NaCl}, 0.1 \%$ Lubrol PX, supplemented with $1 \%$ bovine serum albumin (BSA) and sterile filtered through a $0.22-\mu \mathrm{m}$ filter. If the experiment involves $\mathrm{G} \alpha$ subunits, supplement this buffer with $10 \mu \mathrm{M}$ GDP immediately before use. Store in 50-ml aliquots up to 6 months at $-20^{\circ} \mathrm{C}$

The BSA and detergent concentrations have been optimized to reduce nonspecific binding

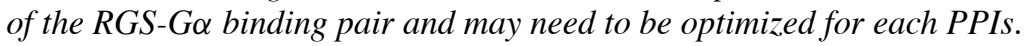

\section{COMMENTARY}

\section{Background Information}

A number of methods have been developed to quantitatively assess the binding of two proteins. The FCPIA approach is generally applicable to the study of PPIs. We have tailored this approach to monitor the interaction between a wide array of RGS proteins and $\mathrm{G} \alpha$ subunits; however, we and our collaborators have successfully used this method to study the interaction between a variety of different proteins. This methodology is quite malleable and can be adapted to the study of many PPIs. To date, it has been applied to relatively high affinity interactions with $K_{\mathrm{d}}$ values ranging from $100 \mathrm{pM}$ to over $300 \mathrm{nM}$. Furthermore, this assay is directly adaptable to high-throughput analysis in a 384-well format. This has been used successfully for small-scale HTS (Feng et al., 2005; Roman et al., 2007) and has been scaled up to large HTS screening campaigns (R. Neubig, unpub. observ. and MLPCN screening IDs: 1423, 1415, 143941). This methodology has some particular advantages over standard solution-phase screening methods (e.g., FP, FRET). First and foremost, this method reduces the effect of spectrally interfering compounds or contaminants by virtue of the analysis technique. Also, the large amount of carrier protein (BSA) and detergent that is used in the assay buffer to reduce nonspecific binding will also minimize the effects of nonspecific aggregators that have been shown, in certain cases, to constitute a large number of the false positives identified in biochemical screens for small molecule protein-protein interaction inhibitors (SMPPIIs) (Feng et al., 2005; Shoichet, 2006).

Another important advantage is the ability to perform multiplexed binding experiments. In this approach, two or more target proteins are assayed for their ability to bind the same reporter protein under conditions of negligible competition between target proteins for free ligand. Thus, multiplexing increases experimental efficiency by reducing reagent/time use and increasing the amount of data generated per experiment. Furthermore, since the binding conditions are truly identical for each target protein, differences in affinity of the targets can be more accurately determined. Using our RGS-G $\alpha$ interaction example, we have multiplexed up to seven different RGS proteins in a single experiment, although theoretically it should be possible to go significantly higher.

These advantages can be combined to provide a convenient method for high-throughput analysis in a multiplexed format. As mentioned previously, we have used this method to perform several high-throughput screens to identify small molecule RGS-G $\alpha$ inhibitors. In these assays, we screened the library against five different RGS proteins simultaneously, thus reducing time, reagent, and compound library use by five-fold.

There are two major limitations to the FCPIA assay. The first limitation is that FCPIA has an affinity limitation that can restrict its use for the characterization of low-affinity interactions. PPIs with low affinities $(>1 \mu \mathrm{M}$ $K_{\mathrm{d}}$ ) tend to be difficult to resolve in this assay,
Multiplexed and MicroparticleBased Analyses

\subsubsection{3}


most likely due to the increased nonspecific adsorption of the reporter protein to the bead. This is not a problem with a homogeneous solution phase PPI assay, where there is no solid substrate for adsorption. The second major limitation to the FCPIA assay is the potential for nonspecific binding that has to be overcome in the assay. For soluble, well-behaved proteins, this limitation is not often an issue. For "stickier" proteins, this limitation is often circumvented by optimizing conditions with a small detergent/blocking agent (e.g., BSA) screen. Other ways to optimize the signal-tonoise of particularly troublesome PPI pairs can be found in the next section. Even with these limitations, the ability to dramatically increase data throughput by assay multiplexing makes FCPIA an attractive complement to the more traditional solution phase protein-protein interaction assays.

\section{Critical Parameters and Troubleshooting}

\section{Nonspecific binding}

If the proper precautions are not taken, there can be significant issues with nonspecific binding in this assay. As with all biochemical assays, the most specific signal from recombinant proteins will be obtained if the target protein is purified to homogeneity before use. The inclusion of up to $1 \% \mathrm{BSA}$ in the assay buffer and detergent (e.g., 0.1\% Lubrol $\mathrm{PX}$ ) was able to minimize nonspecific binding under most circumstances tested thus far. Determining the optimal concentrations and types of carrier proteins and detergents may be PPI specific. A further mechanism to minimize nonspecific fluorescence events in the assay is to ensure that the labeled protein is properly folded and purified away from free label. Biochemical labeling of purified proteins with bulky fluor groups can sometimes cause protein aggregation or misfolding. These improperly folded proteins can contribute significantly to the nonspecific binding signal observed. Optimization of the labeling procedure can minimize these effects, but it is advisable to purify the labeled protein from the reaction mixture using a size-exclusion column to remove both free label and aggregated protein. Other commonly used methods (e.g., spin column, concentration/dilution, dialysis) will not remove aggregated protein and are likely to be less efficient at removing free label.

Low-affinity complexes: The FCPIA approach, unlike some solution-state experiments, has an affinity $\left(K_{\mathrm{d}}\right)$ limitation on the order of $1 \mu \mathrm{M}$. This is likely to be primarily a product of the nonspecific binding that can be observed.

\section{Anticipated Results}

When performed correctly, this method will provide a robust specific binding signal, often 5- to 50-fold above background. In many cases, PPIs are high affinity $\left(K_{\mathrm{d}} \sim \mathrm{nM}\right)$ and they will be easily observed in a quantitative manner. Unlike most assays, saturation binding experiments performed in FCPIA are capable of being multiplexed. While in most fluidphase assays the amount of "receptor" protein required for adequate signal detection is at or just under the expected $K_{\mathrm{d}}$ value of the interaction, the FCPIA approach requires significantly less. In the protocols described above, the binding capacity of the beads limits the amount of target protein to the pM-fM range, which is well below the $K_{\mathrm{d}}$ values observed for most PPIs (nM- $\mu \mathrm{M})$. This provides the advantage that, even at the low concentrations of the reporter protein (e.g., the fluorescently labeled protein), there is minimal competition between different "receptor" proteins in the well. Using this method it is possible to compare the binding of two or more proteins to a "ligand" protein in the same well, saving both time and reagents.

\section{Time Considerations}

\section{Flow cytometry protein interaction saturation assay}

Bead labeling/washing should take $\sim 45$ min. Plate set up and incubation of RGSlabeled beads with fluorescent $\mathrm{G} \alpha_{\mathrm{o}}$ should take an additional $40 \mathrm{~min}$. Instrument set up and data collection on a full 96-well plate in the Luminex 200 should take $\sim 30 \mathrm{~min}$.

\section{Flow cytometry protein interaction competition assay}

Bead labeling/washing should take $\sim 45$ min. Plate set up and incubation of RGS-labeled beads with competitor should take $20 \mathrm{~min}$. Then, an additional $30 \mathrm{~min}$ is required for incubation of the RGS/competitor mixture with fluorescent $\mathrm{G} \alpha_{0}$. Instrument set up and data collection on a full 96-well plate in the Luminex 200 should take $\sim 30 \mathrm{~min}$.

\section{Acknowledgements}

The authors would like to thank Roger Sunahara and John Tesmer at the University of Michigan for helpful discussion. We also 
thank Martha Larsen of the Center for Chemical Genomics for assistance in the development and application of the high-throughput screening methodology. We would also like to thank Larry Sklar and Yang Wu at the University of New Mexico Center for Molecular Discovery for running the multiplex RGS inhibitor screen. We would like to acknowledge the support of the Michigan Chemistry-Biology Interface Training Program, which is funded through the National Institutes of Health under grant number T32 GM00008597. This work was also supported by GM39561 (RRN) and GM076821 (DLR).

\section{Literature Cited}

Berman, D.M., Kozasa, T., and Gilman, A.G. 1996. The GTPase-activating protein RGS4 stabilizes the transition state for nucleotide hydrolysis. $J$. Biol. Chem. 271:27209-27212.

Blazer, L.L. and Neubig, R.R. 2008. Small molecule protein-protein interaction inhibitors as CNS therapeutic agents: Current progress and future hurdles. Neuropsychopharmacology 34:126-141.

Buranda, T., Wu, Y., and Sklar, L.A. 2009. Chapter 11. Subsecond analyses of G-protein coupledreceptor ternary complex dynamics by rapid mix flow cytometry. Methods Enzymol. 461:227247.

Feng, B.Y., Shelat, A., Doman, T.N., Guy, R.K., and Shoichet, B.K. 2005. High-throughput assays for promiscuous inhibitors. Nat. Chem. Biol. 1:146-148.

Jeanneteau, F., Diaz, J., Sokoloff, P., and Griffon, N. 2004a. Interactions of GIPC with dopamine D2, D3 but not D4 receptors define a novel mode of regulation of G protein-coupled receptors. Mol. Biol. Cell 15:696-705.

Jeanneteau, F., Guillin, O., Diaz, J., Griffon, N., and Sokoloff, P. 2004b. GIPC recruits GAIP (RGS19) to attenuate dopamine $\mathrm{D} 2$ receptor signaling. Mol. Biol. Cell 15:4926-4937.

Lee, E., Linder, M.E., and Gilman, A.G. 1994. Expression of G-protein alpha subunits in Escherichia coli. Methods Enzymol. 237:146164.
Neubig, R.R. and Siderovski, D.P. 2002. Regulators of G-protein signalling as new central nervous system drug targets. Nat. Rev. Drug Discov. 1:187-197.

Roman, D.L., Talbot, J.N., Roof, R.A., Sunahara, R.K., Traynor, J.R., and Neubig, R.R. 2007. Identification of small-molecule inhibitors of RGS4 using a high-throughput flow cytometry protein interaction assay. Mol. Pharmacol. 71:169-175.

Roman, D.L., Ota, S., and Neubig, R.R. 2009. Polyplexed Flow Cytometry Protein Interaction Assay: A Novel High-Throughput Screening Paradigm for RGS Protein Inhibitors. J. Biomol. Screen. 14:610-619.

Roof, R.A., Jin, Y., Roman, D.L., Sunahara, R.K., Ishii, M., Mosberg, H.I., and Neubig, R.R. 2006. Mechanism of action and structural requirements of constrained peptide inhibitors of RGS proteins. Chem. Biol. Drug Des. 67:266-274.

Roof, R.A., Sobczyk-Kojiro, K., Turbiak, A.J., Roman, D.L., Pogozheva, I.D., Blazer, L.L., Neubig, R.R., and Mosberg, H.I. 2008. Novel peptide ligands of RGS4 from a focused onebead, one-compound library. Chem. Biol. Drug Des. 72:111-119.

Sarvazyan, N.A., Remmers, A.E., and Neubig, R.R. 1998. Determinants of gilalpha and beta gamma binding. Measuring high affinity interactions in a lipid environment using flow cytometry. J. Biol. Chem. 273:7934-7940.

Shankaranarayanan, A., Thal, D.M., Tesmer, V.M., Roman, D.L., Neubig, R.R., Kozasa, T., and Tesmer, J.J. 2008. Assembly of high order G alpha q-effector complexes with RGS proteins. J. Biol. Chem. 283:34923-34934.

Shoichet, B.K. 2006. Screening in a spirit haunted world. Drug Discov. Today 11:607-615.

Simons, P.C., Shi, M., Foutz, T., Cimino, D.F., Lewis, J., Buranda, T., Lim, W.K., Neubig, R.R., McIntire, W.E., Garrison, J., Prossnitz, E., and Sklar, L.A. 2003. Ligand-receptor-G-protein molecular assemblies on beads for mechanistic studies and screening by flow cytometry. Mol. Pharmacol. 64:1227-1238.

Sklar, L.A., Edwards, B.S., Graves, S.W., Nolan, J.P., and Prossnitz, E.R. 2002. Flow cytometric analysis of ligand-receptor interactions and molecular assemblies. Annu. Rev. Biophys. Biomol. Struct. 31:97-119.
Multiplexed and MicroparticleBased Analyses

\subsubsection{5}

